
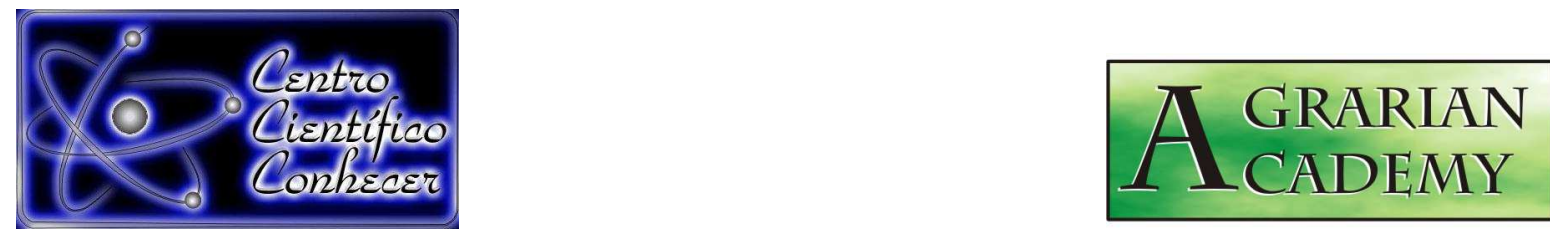

\title{
AJUSTES DE CURVAS DE CRESCIMENTO DE PESO SIMULADOS DE CAPRINOS DE DIFERENTES RAÇAS
}

\author{
Leandro Ricardo Rodrigues de Lucena
}

Prof. Dr. Departamento de Zootecnia, Universidade Federal Rural de Pernambuco, Serra Talhada, PE, Brasil, leandroricardo_est@yahoo.com.br.

Recebido em: 15/04/2017 - Aprovado em: 22/07/2017 - Publicado em: 31/07/2017 DOI: 10.18677/Agrarian Academy 2017a1

\section{RESUMO}

O estudo do crescimento, por meio de ajustamento de uma função que descreva todo o período de vida do animal, relacionando peso e idade tem sido amplamente discutido nas últimas décadas. O peso de caprinos sem raça definida, mestiços e Anglo-nubiana foram simulados através da distribuição de probabilidade uniforme num intervalo [a,b]. Foram ajustados os modelos de regressão logístico, gompertz, polinomial quadrático e gamma para explicar possível relação do peso/idade dos caprinos. Verificou-se que os modelos polinomial quadrático e gamma apresentaram as melhores estimativas para explicar o comportamento do peso das diferentes raças dos caprinos. O modelo gamma apresentou grau de explicação $R^{2}=99,36 \%$ para caprinos sem raça definida, mestiços $R^{2}=99,81 \%$ e Anglo-nubiana $R^{2}=$ $99,77 \%$, enquanto que o modelo polinomial quadrático apresentou $R^{2}=99,52 \%$ para caprinos sem raça definida, $\mathrm{R}^{2}=99,84 \%$ para mestiços e Anglo-nubiana de $\mathrm{R}^{2}=$ $99,84 \%$. Optou-se pelo modelo gamma para explicar o comportamento do peso dos caprinos independente da raça avaliada, pois o mesmo apresentou alto poder de explicação e valores pequenos de critério de informação de Akaike e de soma de quadrados dos resíduos, além de apresentar um parâmetro a menos que o modelo quadrático.

PALAVRAS-CHAVE: curvas de crescimento; caprinos; regressão não-linear.

\section{ADJUSTMENTS OF GROWTH CURVE OF SIMULATED WEIGHT GOATS OF DIFFERENT BREEDS}

\footnotetext{
ABSTRACT

The study of growth, by adjusting a function that describes the whole life period of animal, relating weight and age has been widely discussed in last decades. The weight of undefined, mestizo and Anglo-Nubian goats were simulated through the uniform probability distribution over a range [a, b]. Logistic, Gompertz, quadratic polynomial and gamma regression models were adjusted to explain possible weight / age relation of goats. It was verified that the polynomial quadratic and gamma models presented the best estimates to explain the weight behavior of different goat breeds. The gamma model presented a degree of explanation $\left(R^{2}=99.36 \%\right)$ for AGRARIAN ACADEMY, Centro Científico Conhecer - Goiânia, v.4, n.7; p. 12017
} 
undefined goats, mestizos $\left(R^{2}=99.81 \%\right)$ and Anglo-Nubian $\left(R^{2}=99.77 \%\right)$, while the quadratic polynomial model presented $R^{2}=99.52 \%$ for undefined goats, $R^{2}=99.84 \%$ for mestizos and Anglo-Nubian $\mathrm{R}^{2}=99.84 \%$. The gamma model was used to explain the behavior of goat weight independently of breed evaluated, since it had high explanatory power and small values of Akaike Information Criterion and sum of squares of residues, besides of presenting a parameter a Less than the quadratic model.

KEYWORDS: Growth curve; Goats; nonlinear regression.

\section{INTRODUÇÃO}

O estudo do crescimento, por meio de ajustamento de uma função que descreva todo o período de vida do animal, relacionando peso e idade tem sido pesquisado por vários autores: MACEDO et al., (2014), MORAIS et al., (2015), TEXEIRA et al., (2012). Análises do crescimento animal são de fundamental importância na produção animal, pois são analisados ao longo de diversas condições ambientais tais como: tempo, clima e umidade do ar (FREITAS, 2005).

O conhecimento das curvas de crescimento de uma espécie se faz necessário, pois fornece informações muito úteis para o cultivo e manejo, além de ter grande importância biológica. A curva descrevendo uma sequência de medidas de determinada característica de alguma espécie ou indivíduo em função do tempo, geralmente peso, altura ou diâmetro, é chamada de curva de crescimento (FERNANDES et al., 2012). Este tipo de curva em geral apresenta um crescimento em formato de "S" (crescimento exponencial ou sigmoidal), que é muito bem modelado pela regressão não linear (FERNANDES et al., 2012).

Estudos sobre curvas de crescimentos em animais vêm sendo bastante explorados por vários autores, na avicultura destacam-se os trabalhos de: VARGAS et al., (2006), MORAIS et al., (2015). Na bovinocultura os estudos SILVA et al., (2011) e SOUZA et al., (2010) merecem destaque. Na ovinocultura pode-se relatar os trabalhos de: TEXEIRA et al., (2012), Ó et al., (2012), GBANGBOCHE et al., (2008). Na suinocultura destacam-se os estudos de: DUTRA Jr. et al., (2001), FREITAS, (2005), OLIVEIRA et al., (2007), MACEDO et al., (2014).

$\mathrm{Na}$ caprinocultura os trabalhos de MALHADO et al., (2008) com caprinos da raça Anglo-Nubiana criados na caatinga, CARNEIRO et al., (2009) com caprinos da raça Mambrina e FIGUEIREDO FILHO et al., (2012) com caprinos mestiços verificaram que os modelos de Von Bertalanffy, Gompertz e Logístico apresentaram os melhores ajustes para descrever o peso dos caprinos. CRUZ et al., (2009) em estudo com caprinos mestiços no estado da Paraíba observaram que o modelo quadrático logarítmico foi o mais adequado para explicar o crescimento dos caprinos. CAVALCANTE et al., (2013) demonstraram em estudo que o modelo mais adequado para explicar o crescimento de caprinos sem raça definida foi o logístico, enquanto que SOUZA et al., (2011) verificaram que os modelos de Von Bertalanffy, Brody e Richards foram os mais adequados para explicar o peso dos caprinos da raça morada nova criados no estado da Bahia. GADDOUR et al., (2012) em estudo notaram que o modelo de gompertz foi o mais adequado para explicar o crescimento do peso de caprinos, o mesmo foi observado por WAHEED et al., (2011) com caprinos da raça Beetal, já OZDEMIR \& DELLAL (2009) definiram que os modelos logístico e gompertz foram os mais adequados para explicar o crescimento de caprinos da raça Angorá. 
Os autores supracitados não chegaram a um consenso de qual o melhor modelo para explicar o comportamento do peso de caprinos levando em consideração as raças. Diante disso este trabalho objetivou modelar o crescimento de peso simulado de caprinos através de uma série de modelos de regressão (lineares, não lineares e polinomiais).

\section{MATERIAL E MÉTODOS}

\section{Dados}

Foram simulados valores de peso para caprinos das raças: sem raça definida (SRD), mestiços, Anglo-Nubiana, no período de 0 (ao nascimento) a 147 dias, em intervalos de sete em sete dias de mensuração. Para cada período de tempo foram simuladas 10 observações de uma variável aleatória com distribuição uniforme contínua e a média calculada.

Seja $X$ uma variável com distribuição de probabilidade uniforme num intervalo [a, b], então definiu-se a função de densidade de probabilidade de $\mathrm{X}$ por:

$$
\begin{gathered}
f(x)=1 /(b-a) ; \text { se } a \leq x \leq b \\
0 ; \text { caso contrário }
\end{gathered}
$$

Para cada período de tempo foram selecionados de forma aleatória cinco observações da distribuição uniforme definida no intervalo [a, b] e depois calculado a média desses valores utilizando a fórmula abaixo:

$$
x=a+(b-a) R
$$

onde, a e b são o menor e o maior valor do peso do suíno para uma determinada idade e R é um valor aleatório entre 0 e 1, assim definiu-se os valores de pesos simulados.

Os intervalos de pesos em relação aos dias e as diferentes raças de caprinos estão dispostos na Tabela 1 e os valores simulados dos pesos dos caprinos das diferentes raças estão dispostos na Figura 1.

TABELA 1. Intervalos dos pesos $(\mathrm{kg})[\mathrm{a} ; \mathrm{b}]$ dos caprinos das diferentes raças.

\begin{tabular}{lccc}
\hline \multirow{2}{*}{ Período de avaliação } & \multicolumn{3}{c}{ Intervalos dos pesos (kg) [a;b] } \\
& SDR & Mestiços & $\begin{array}{c}\text { Anglo- } \\
\text { nubiana }\end{array}$ \\
\hline 0 & {$[1,5 ; 2,2]$} & {$[2,5 ; 3,2]$} & {$[2,2 ; 2,6]$} \\
7 & {$[2,5 ; 3,0]$} & {$[3,5 ; 3,9]$} & {$[2,7 ; 3,1]$} \\
14 & {$[3,3 ; 3,9]$} & {$[4,0 ; 4,7]$} & {$[3,2 ; 3,7]$} \\
21 & {$[4,2 ; 4,7]$} & {$[4,8 ; 5,3]$} & {$[3,8 ; 4,2]$} \\
28 & {$[4,8 ; 5,1]$} & {$[5,4 ; 6,0]$} & {$[4,4 ; 5,0]$} \\
35 & {$[5,2 ; 5,5]$} & {$[6,1 ; 6,4]$} & {$[5,1 ; 5,5]$} \\
42 & {$[5,6 ; 6,0]$} & {$[6,5 ; 6,8]$} & {$[5,7 ; 6,1]$} \\
49 & {$[6,2 ; 6,6]$} & {$[6,9 ; 7,3]$} & {$[6,3 ; 6,7]$} \\
56 & {$[6,8 ; 7,2]$} & {$[7,4 ; 7,9]$} & {$[6,9 ; 7,3]$} \\
63 & {$[7,2 ; 7,5]$} & {$[8,1 ; 8,5]$} & {$[7,3 ; 7,7]$} \\
70 & {$[7,5 ; 7,9]$} & {$[8,6 ; 9,0]$} & {$[7,8 ; 8,3]$} \\
77 & {$[8,3 ; 8,7]$} & {$[9,2 ; 9,5]$} & {$[8,4 ; 8,7]$} \\
84 & {$[8,8 ; 9,1]$} & {$[9,5 ; 10,0]$} & {$[9,0 ; 9,5]$} \\
91 & {$[9,3 ; 9,6]$} & {$[10,5 ; 10,9]$} & {$[10,0 ; 10,5]$} \\
98 & {$[9,8 ; 10,2]$} & {$[11,0 ; 11,5]$} & {$[10,7 ; 11,1]$}
\end{tabular}

AGRARIAN ACADEMY, Centro Científico Conhecer - Goiânia, v.4, n.7; p. 32017 


\begin{tabular}{llll}
105 & {$[10,5 ; 10,9]$} & {$[11,7 ; 12,1]$} & {$[11,3 ; 11,7]$} \\
112 & {$[11,3 ; 11,8]$} & {$[12,3 ; 12,7]$} & {$[12,0 ; 12,4]$} \\
119 & {$[12,0 ; 12,5]$} & {$[12,9 ; 13,5]$} & {$[12,5 ; 13,0]$} \\
126 & {$[12,7 ; 13,2]$} & {$[13,7 ; 14,0]$} & {$[13,1 ; 13,5]$} \\
133 & {$[13,5 ; 14,0]$} & {$[14,2 ; 14,6]$} & {$[13,6 ; 14,0]$} \\
140 & {$[14,1 ; 14,5]$} & {$[14,8 ; 15,1]$} & {$[14,2 ; 14,6]$} \\
147 & {$[14,7 ; 15,1]$} & {$[15,2 ; 15,7]$} & {$[14,7 ; 15,2]$} \\
\hline
\end{tabular}

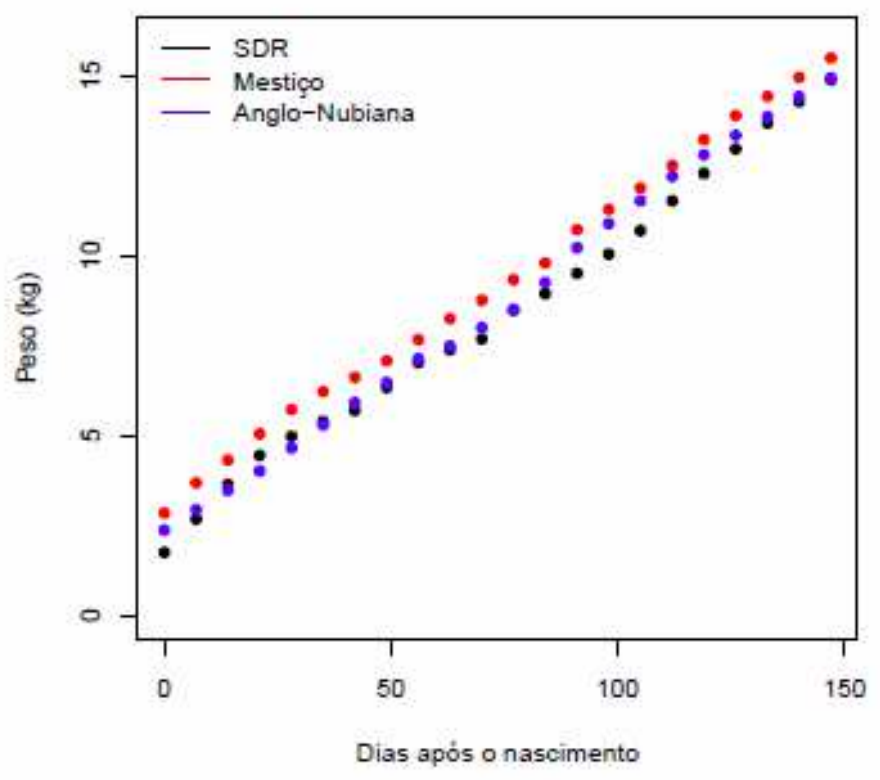

FIGURA 1. Valores simulados dos pesos dos caprinos em relação as raças e o tempo de aferição

\section{Modelos de regressão}

Para avaliar a relação entre o ganho de peso dos caprinos sem raça definida, mestiços e Anglo-nubiana e a idade dos mesmos, foram escolhidos vários modelos de regressão para explicar tal relação. Os modelos utilizados para avaliar a relação do peso/idade foram os seguintes: logístico, gompertz, gamma e polinomial quadrático.

\section{Modelo logístico}

Seja X uma variável aleatória com distribuição logística, define-se a função de densidade de probabilidade em (1).

$$
f(x)=\omega \beta \exp (\alpha+\beta X) /(\exp (\alpha)+\exp (\beta X))^{2}
$$

então, determina-se o modelo logístico por:

$$
Y_{i}=\omega /\left(1+\exp \left(\beta_{0}+\beta_{1} \text { dias }_{i}\right)\right)+\varepsilon_{i} \quad i=1,2,3, \ldots, n
$$

onde, $Y_{i}$ é o peso simulado do i-ésimo caprino após o nascimento; dias $s_{i}$ é o i-ésimo dia de avaliação e $\varepsilon_{i}$ é o i-ésimo erro associado ao peso do caprino, em que $\varepsilon_{i}$ 
apresenta distribuição normal de média 0 e variância constante $\sigma^{2}$. As incógnitas $\omega$, $\beta_{0}$ e $\beta_{1}$ são os parâmetros associados ao modelo.

\section{Modelo gompertz}

Seja X uma variável aleatória com distribuição Gompertz, defini-se a função de densidade de probabilidade em (3).

$$
f(x)=\omega \beta \exp (-\exp (\alpha-\beta X))
$$

então denota-se o modelo gompertz por:

$$
Y_{i}=\omega \exp \left(-\exp \left(\beta_{0}+\beta_{1} \text { dias }_{i}\right)\right)+\varepsilon_{i} \quad i=1,2,3, \ldots, n
$$

onde, $Y_{i}$ é o peso simulado do i-ésimo caprino após o nascimento; dias $s_{i}$ é o i-ésimo dia de avaliação após o nascimento do caprino e $\varepsilon_{i}$ é o i-ésimo erro associado ao peso do caprino, em que $\varepsilon_{i}$ apresenta distribuição normal de média 0 e variância constante $\sigma^{2}$. As incógnitas $\omega, \beta_{0}$ e $\beta_{1}$ são os parâmetros associados ao modelo.

\section{Modelo polinomial}

O modelo de regressão polinomial quadrático é definido por (5).

$$
Y_{i}=\beta_{0}+\beta_{1} \operatorname{dias}_{i}+\beta_{2}\left(\text { dias }_{i}\right)^{2}+\varepsilon_{i} \quad i=1,2,3, \ldots, 7(5)
$$

onde, $Y_{i}$ é o peso simulado do i-ésimo caprino após o nascimento; dias $s_{i}$ é o i-ésimo dia de avaliação e $\varepsilon_{i}$ é o i-ésimo erro associado ao peso, em que $\varepsilon_{\mathrm{i}}$ apresenta distribuição normal de média 0 e variância constante $\sigma^{2}$. As incógnitas $\beta_{0}, \beta_{1}$ e $\beta_{2}$ são os parâmetros associados ao modelo.

\section{Modelo gamma}

Seja $X$ uma variável aleatória com distribuição gamma, define-se a função de densidade de probabilidade em (6).

$$
f(x)=1 /\left(\Gamma(\alpha) \beta^{\alpha}\right) x^{\alpha-1} \exp (-x / \beta)
$$

logo, determina-se o modelo gamma com função de ligação identidade por:

$$
\mathrm{Yi}=\beta_{0}+\beta_{1} \text { dias }_{i}+\varepsilon_{i} \quad \mathrm{i}=1,2,3, \ldots, \mathrm{n}(7)
$$

onde, Yi é o peso simulado do i-ésimo caprino após o nascimento; diasi é o i-ésimo dia de avaliação e $\varepsilon_{\mathrm{i}}$ é o i-ésimo erro associado ao peso, em que $\varepsilon_{\mathrm{i}}$ apresenta distribuição gamma de parâmetros $\alpha$ e $\beta$. As incógnitas $\beta_{0}$ e $\beta_{1}$ são os parâmetros associados ao modelo.

\section{Critérios de seleção dos modelos}

Os modelos foram avaliados pelos seguintes critérios: maior coeficiente de determinação do modelo $\left(R^{2}\right)$, menor critério de informação de Akaike (AIC) e soma de quadrados dos resíduos (SQR).

AGRARIAN ACADEMY, Centro Científico Conhecer - Goiânia, v.4, n.7; p. 52017 
Seja $\widehat{Y}_{\imath} \circ$ i-ésimo valor do peso dos caprinos após ajuste do modelo, então defini-se a soma dos quadrados dos resíduos para este estudo pela seguinte expressão:

$$
\mathrm{SQR}=\sum_{i=1}^{n}\left(Y_{i}-\widehat{Y}_{i}\right)^{2}
$$

O coeficiente de determinação do modelo $\left(R^{2}\right)$ é expresso pela razão entre a soma de quadrado do modelo (SQM) e a soma de quadrados total (SQT), ou seja,

$$
\mathrm{R}^{2}=\frac{\mathrm{SQM}}{\mathrm{SQT}}=1-\mathrm{SQR} / \mathrm{SQT}=1-\frac{\sum_{i=1}^{n}\left(Y_{i}-\hat{Y}_{V^{2}}\right)^{2}}{\sum_{l=1}^{n}\left(Y_{i}-Y_{l}\right)^{2}}
$$

O critério de informação de Akaike (AIC) definido por AKAIKE (1974) é dado por:

$$
A I C=-2 \ln L(x \mid \hat{\theta})+2(p)
$$

onde, $L(x \mid \hat{\theta})$ é a função de máxima verossimilhança, definida como sendo o produtório da função de densidade e $p$ é quantidade de parâmetros do modelo.

\section{RESULTADOS E DISCUSSÃO}

$\mathrm{Na}$ Figura 2 observa-se que o modelo logístico não apresentou bons ajustes para os pesos simulados dos caprinos das três raças avaliadas.
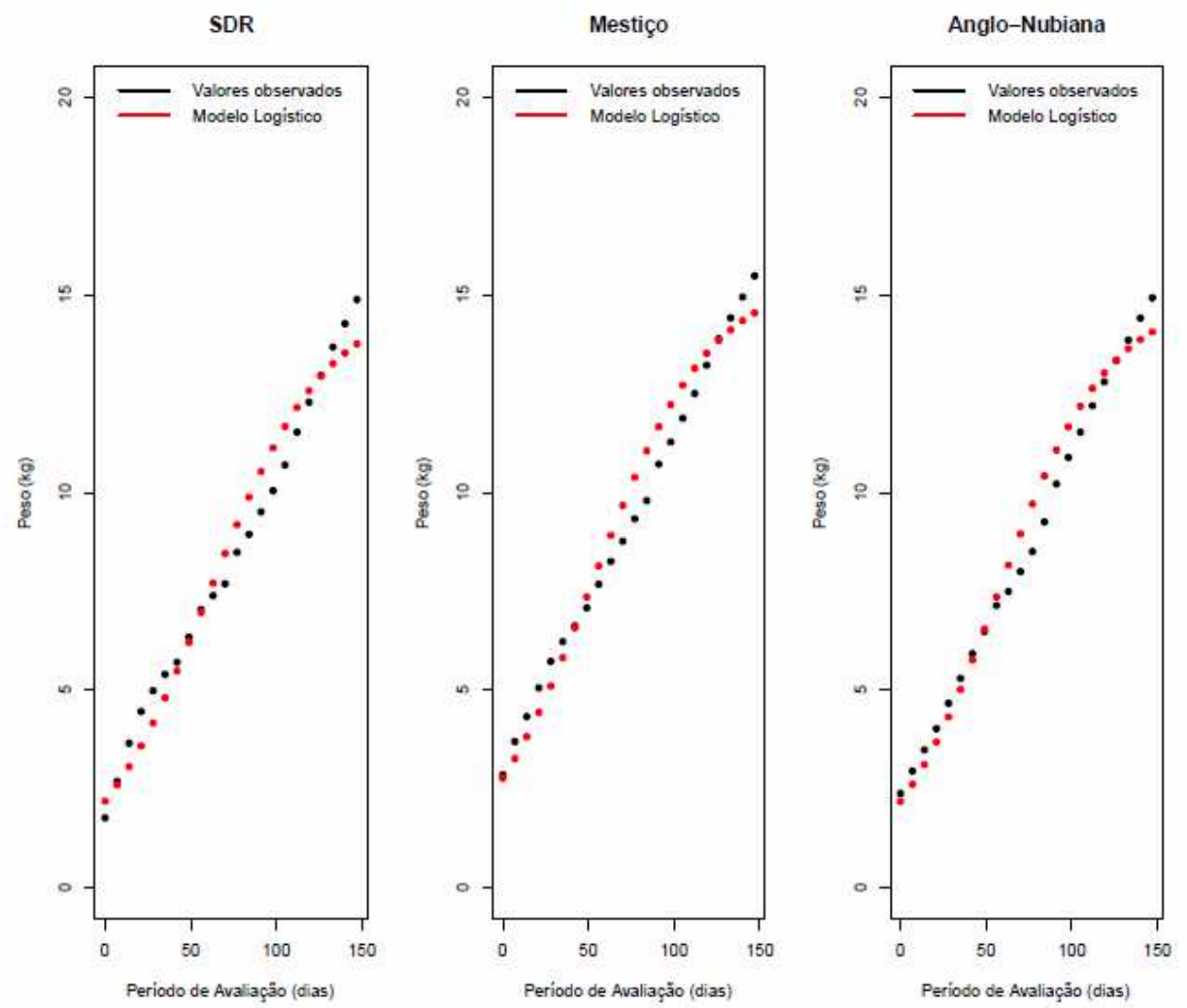

FIGURA 2. Ajuste do modelo logístico para peso simulados dos caprinos das diferentes raças. 
Após ajuste do modelo logístico verificou-se que o grau de explicação, critério de informação de Akaike e a soma dos quadrados dos resíduos para os caprinos SDR foram $R^{2}=94,81 \%, A I C=13,139$ e $S Q R=10,055$, para os mestiços $R^{2}=95$, $15 \%, A I C=11,022$ e $S Q R=9,936$, e anglo-nubiana $R^{2}=96,41 \%, A I C=7,373$ e $S Q R=$ 7,935, ( Tabela 2).

TABELA 2. Modelo logístico ajustado e critérios de adequação do modelo para as diferentes raças de caprinos.

\begin{tabular}{|c|c|c|c|c|}
\hline \multirow{2}{*}{$\begin{array}{l}\text { Raças dos } \\
\text { caprinos }\end{array}$} & \multirow[t]{2}{*}{ Equação do modelo logístico } & \multicolumn{3}{|c|}{$\begin{array}{l}\text { Critérios de Adequação } \\
\text { do Modelo }\end{array}$} \\
\hline & & $\mathrm{R}^{2}$ & SQR & $\mathrm{AIC}$ \\
\hline SDR & $\widetilde{\mathrm{Y}}=14,883 / 1+\exp (1,755-0,029 \mathrm{dias})$ & 94,81 & 10,055 & 13,139 \\
\hline Mestiços & $\widehat{\mathrm{Y}}=15,483 / 1+\exp (1,521-0,029$ dias $)$ & 95,15 & 9,936 & 11,022 \\
\hline $\begin{array}{l}\text { Anglo- } \\
\text { nubiana }\end{array}$ & $\widehat{\mathrm{Y}}=14,927 / 1+\exp (1,765-0,031$ dias $)$ & 96,41 & 7,935 & 7,373 \\
\hline
\end{tabular}

Resultados semelhantes na avaliação do modelo logístico foram apresentados por MALHADO et al., (2008) quando constataram $R^{2}=98,6 \%$ e $\mathrm{SQR}=12,9$ para os caprinos da raça Anglo-Nubiana, já para mestiços o estudo de FIGUEIREDO FILHO et al. (2012) vem a corroborar com os achados desta pesquisa, onde os mesmos apresentaram $R^{2}=98,55$ e $S Q R=16,8$. No estudo de CAVALCANTE et al., (2013) avaliando caprinos sem raça definida apresentaram $R^{2}=99 \%$ e $\mathrm{SQR}=2,25$, resultados próximos aos desta pesquisa.

Verifica-se na Figura 3 que assim como o modelo logístico o modelo de Gompertz não apresentou bons ajustes para os pesos simulados dos caprinos das três raças avaliadas.
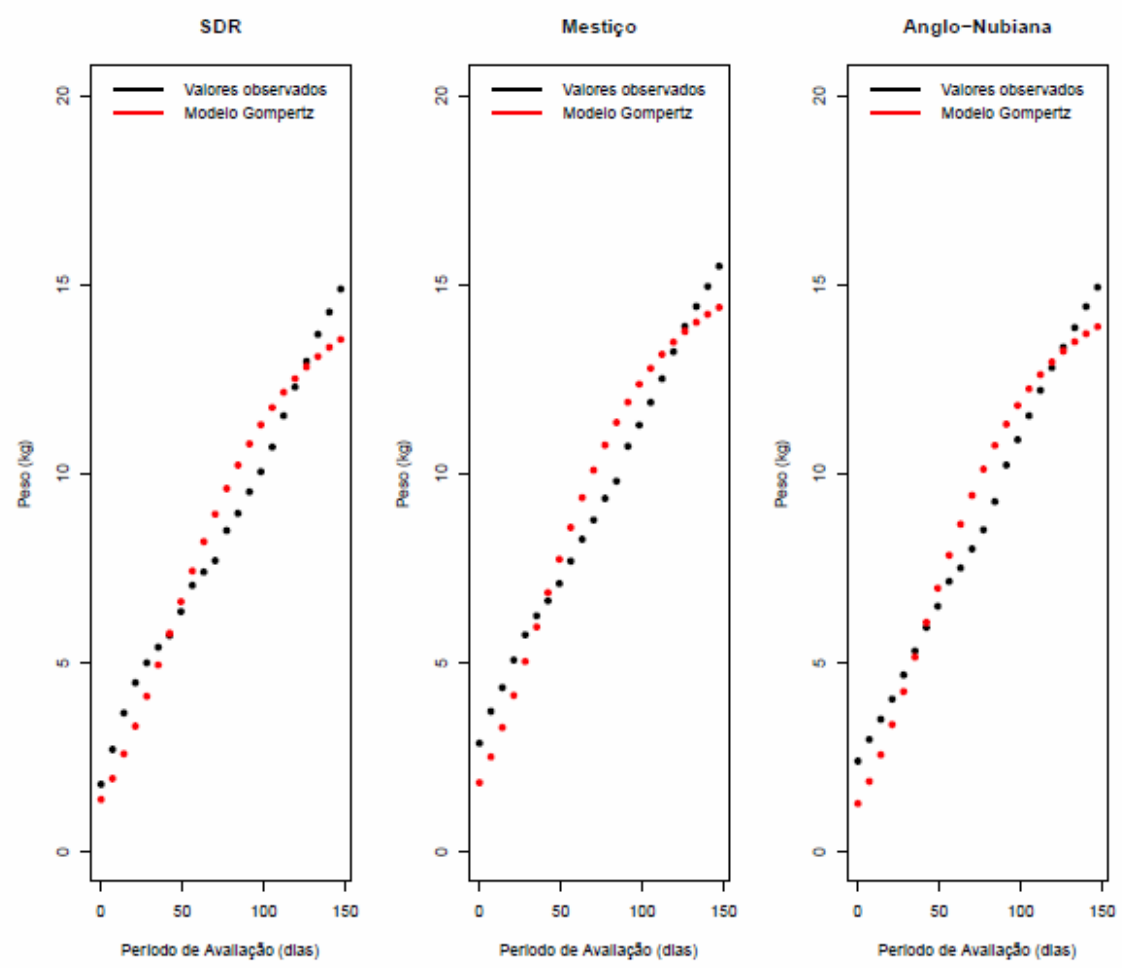

FIGURA 3. Ajuste do modelo gompertz para peso simulados dos caprinos das diferentes raças. 
O modelo de gompertz apresentou grau de explicação $\left(R^{2}=90,4 \%\right)$ para os caprinos SDR, $\left(R^{2}=90,8 \%\right)$ para os mestiços e $\left(R^{2}=91,99 \%\right)$ para Anglo-nubiana. Para os caprinos SDR o modelo de Gompertz apresentou AIC=15,578 e $\mathrm{SQR}=17,203$, mestiços $\mathrm{AIC}=15,722$ e $\mathrm{SQR}=19,499$ e para os caprinos da raça Anglo-nubiana $\mathrm{AIC}=14,608$ e SQR=17,354, (Tabela 3).

TABELA 3. Modelo gompertz ajustado e critérios de adequação do modelo para as diferentes raças de caprinos.

\begin{tabular}{llccc} 
& & \multicolumn{3}{c}{ Critérios de Adequação } \\
\cline { 3 - 5 } $\begin{array}{l}\text { Raças dos } \\
\text { caprinos }\end{array}$ & Equação do modelo gompertz & \multicolumn{3}{c}{ do Modelo } \\
\cline { 3 - 5 } SDR & $\tilde{\mathrm{Y}}=14,883 \exp (-\exp (0,87-0,022$ dias)) & 90,40 & 17,203 & AIC \\
Mestiços & $\tilde{\mathrm{Y}}=15,483 \exp (-\exp (0,763-0,023$ dias)) & 90,80 & 19,499 & 15,722 \\
$\begin{array}{l}\text { Anglo- } \\
\text { nubiana }\end{array}$ & $\hat{\mathrm{Y}}=14,927 \exp (-\exp (0,905-0,024$ dias)) & 91,99 & 17,354 & 14,608 \\
\hline
\end{tabular}

Resultados semelhantes na avaliação do modelo Gompertz foram apresentados por MALHADO et al., (2008) quando observaram $R^{2}=98,7 \%$ e SQR $=11,8$ para os caprinos da raça Anglo-Nubiana, já para mestiços o estudo de FIGUEIREDO FILHO et al. (2012) vem a corroborar com os achados desta pesquisa, onde os mesmos apresentaram $R^{2}=98,42 \%$ e $S Q R=7,70$.

$\mathrm{Na}$ Figura 4 verifica-se que 0 modelo quadrático apresentou ótimas estimativas dos pesos simulados dos caprinos sem raça definida, mestiços e Anglonubiana, respectivamente para todo período de avaliação.
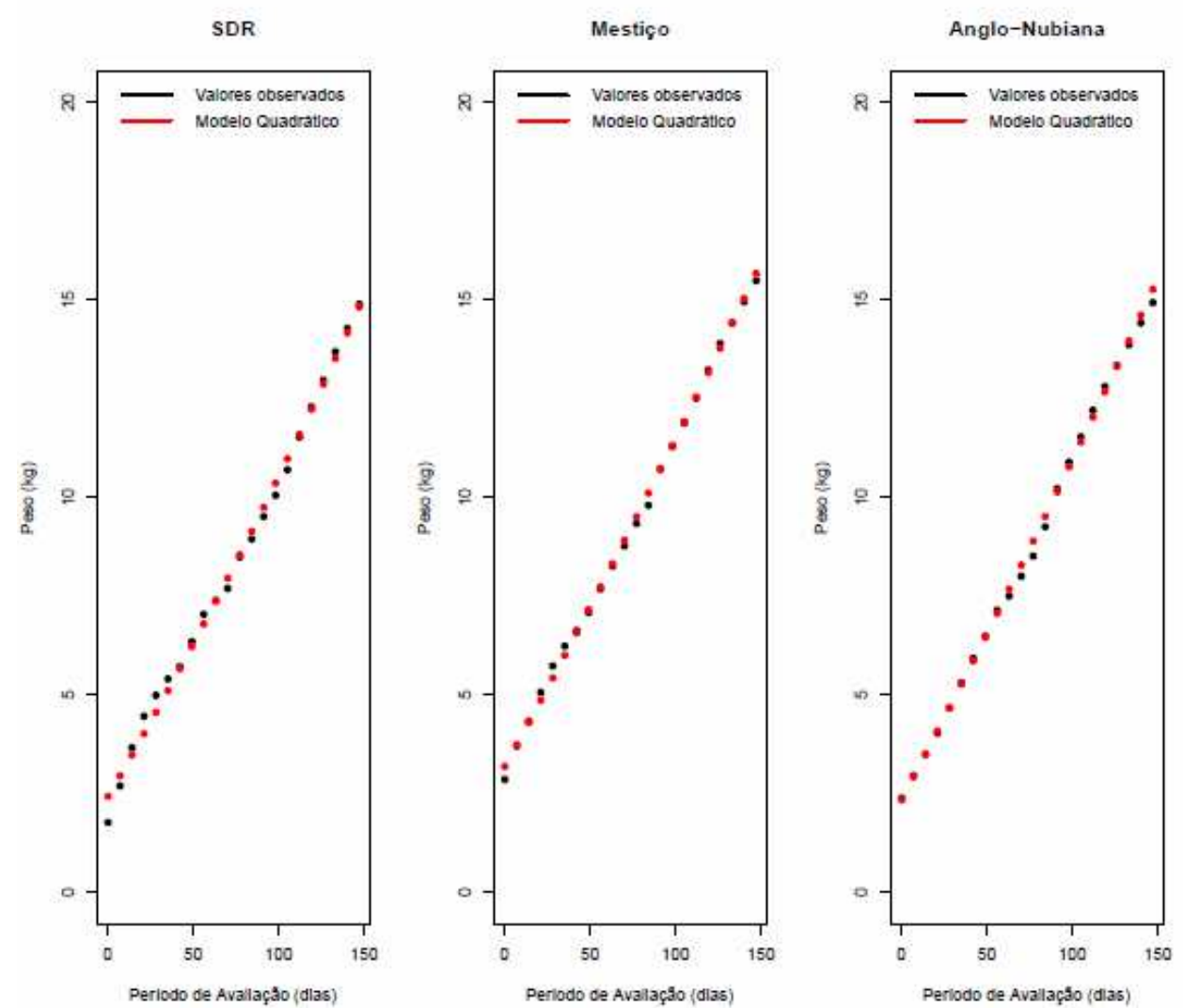

FIGURA 4. Ajuste do modelo quadrático para peso simulados dos caprinos das diferentes raças.

AGRARIAN ACADEMY, Centro Científico Conhecer - Goiânia, v.4, n.7; p. 82017 
O modelo quadrático apresentou alto poder de explicação para as três raças avaliadas, SDR $\left(R^{2}=99,52 \%\right)$, mestiços $\left(R^{2}=99,84 \%\right)$ e Anglo-nubiana $\left(R^{2}=99\right.$, 84\%). O modelo quadrático além de apresentar alto poder de explicação apresentou baixo critério de informação de Akaike e soma de quadrados dos resíduos para as três raças avaliadas, SDR (AIC=10,947 e $S Q R=1,473)$, mestiços $(A I C=-13,266$ e $\mathrm{SQR}=0,512$ ) e Anglo-nubiana ( $\mathrm{AIC}=-11,245$ e $\mathrm{SQR}=0,589$ ), (Tabela 4).

TABELA 4. Modelo quadrático ajustado e critérios de adequação do modelo para as diferentes raças de caprinos.

\begin{tabular}{|c|c|c|c|c|}
\hline \multirow{2}{*}{$\begin{array}{l}\text { Raças dos } \\
\text { caprinos }\end{array}$} & \multirow[t]{2}{*}{ Equação do modelo quadrático } & \multicolumn{3}{|c|}{$\begin{array}{l}\text { Critérios de Adequação } \\
\text { do Modelo }\end{array}$} \\
\hline & & $\mathrm{R}^{2}$ & SQR & $\mathrm{AIC}$ \\
\hline SDR & $\widehat{\mathrm{Y}}=2,427+0,074$ dias $+0,00007$ dias $^{2}$ & 99,52 & 1,473 & 10,947 \\
\hline Mestiços & $\widehat{\mathrm{Y}}=3,184+0,079$ dias $+0,00004$ dias $^{2}$ & 99,84 & 0,512 & $-13,266$ \\
\hline $\begin{array}{l}\text { Anglo- } \\
\text { nubiana }\end{array}$ & $\widehat{\mathrm{Y}}=2,343+0,082$ dias $+0,00004$ dias $^{2}$ & 99,84 & 0,589 & $-11,245$ \\
\hline
\end{tabular}

CRUZ et al., (2009) constataram que para caprinos mestiços o modelo quadrático apresentou $\mathrm{R}^{2}=97,0 \%$, resultado este que corrobora com o estudos em questão.

$\mathrm{Na}$ avaliação do modelo gamma verificou-se que o mesmo apresentou estimativas muito próximas dos valores observados dos pesos dos caprinos em relação as diferentes raças para todo período de avaliação, ( Figura 5).
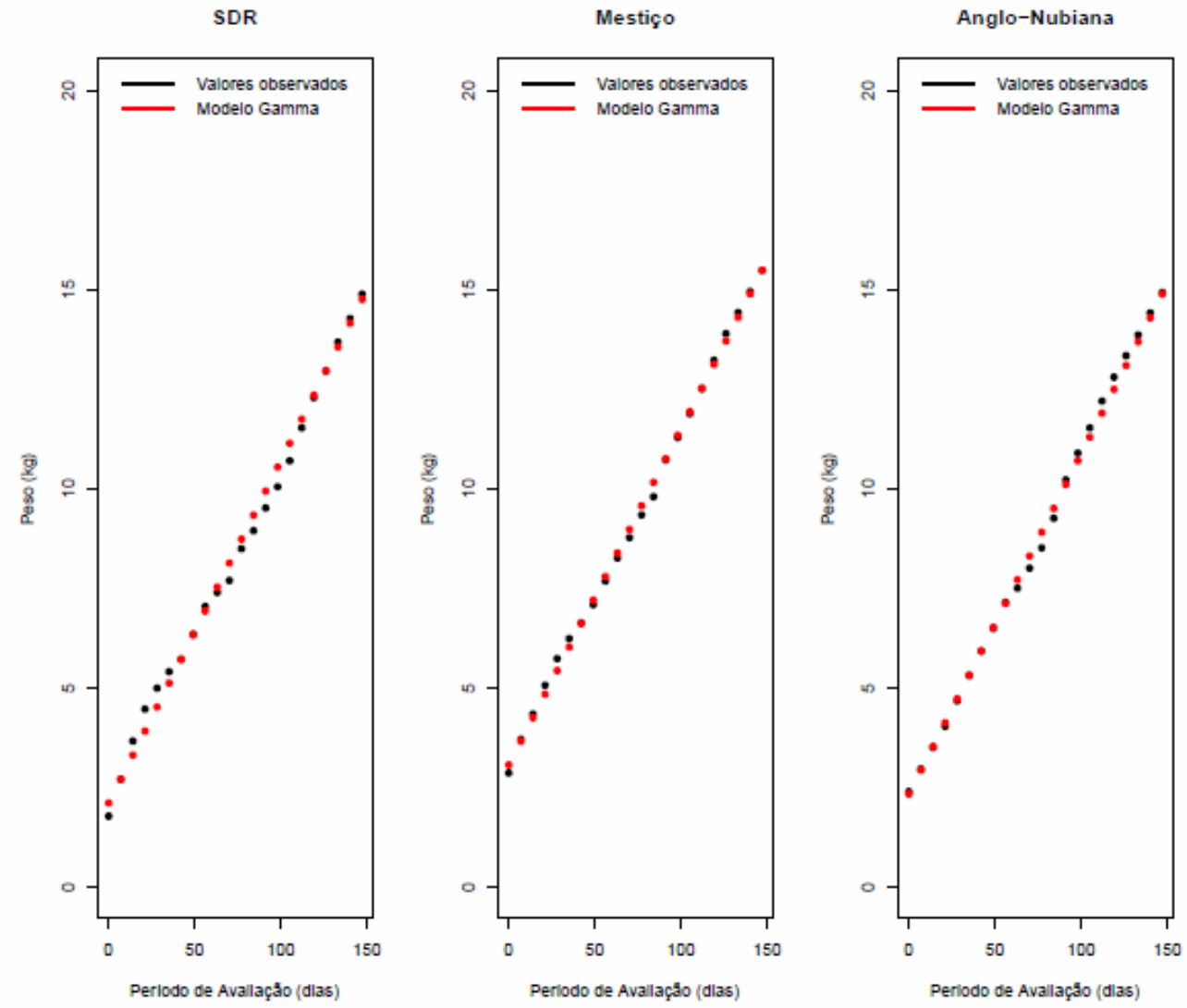

FIGURA 5. Ajuste do modelo gamma para peso simulados dos caprinos das diferentes raças.

AGRARIAN ACADEMY, Centro Científico Conhecer - Goiânia, v.4, n.7; p. 92017 
O modelo gamma apresentou alto poder de explicação para os caprinos SDR $\left(R^{2}=99,36 \%\right)$, mestiços $\left(R^{2}=99,81 \%\right)$ e Anglo-nubiana $\left(R^{2}=99,77 \%\right)$. O modelo gamma apresentou $\mathrm{AIC}=33,44$ e $\mathrm{SQR}=1,99$, para os caprinos $\mathrm{SDR}$, para os mestiços $A I C=1,847$ e $S Q R=0,58$ e Anglo-nubiana: $A I C=-13,548$ e $S Q R=0,775$, (Tabela 5).

TABELA 5. Modelo gamma ajustado e critérios de adequação do modelo para as diferentes raças de caprinos.

\begin{tabular}{llccc}
\hline \multirow{2}{*}{$\begin{array}{l}\text { Raças dos } \\
\text { caprinos }\end{array}$} & $\begin{array}{l}\text { Equação do modelo } \\
\text { gamma }\end{array}$ & \multicolumn{3}{c}{$\begin{array}{c}\text { Critérios de Adequação } \\
\text { do Modelo }\end{array}$} \\
\cline { 3 - 5 } & & $\mathrm{R}^{2}$ & SQR & AIC \\
\hline SDR & $\overline{\mathrm{Y}}=2,100+0,086$ dias & 99,36 & 1,990 & 33,440 \\
Mestiços & $\overline{\mathrm{Y}}=3,059+0,085$ dias & 99,81 & 0,580 & 1,847 \\
$\begin{array}{l}\text { Anglo- } \\
\text { nubiana }\end{array}$ & $\mathrm{Y}=2,326+0,085$ dias & 99,77 & 0,775 & $-13,548$ \\
\hline
\end{tabular}

CRUZ et al. (2009) apresentaram resultados que vem a corroborar com os achados desta pesquisa na avaliação do modelo gamma, onde os mesmo apresentaram grau de explicação de $97,0 \%$, resultados este abaixo do encontrado neste estudo.

Os modelos quadráticos e gamma foram selecionados utilizando os três critérios de seleção como os mais adequados para explicar o crescimento do peso simulado dos caprinos das três raças avaliadas. Optou-se como modelo mais adequado para explicar o crescimento dos caprinos, independente da raça avaliada, o modelo gamma, pois além de apresentar alto grau de explicação e baixos valores de AIC e SQR, apresenta um parâmetro a menos para explicar o comportamento do crescimento do peso dos caprinos quando comparado com o modelo quadrático.

\section{CONCLUSÃO}

O peso simulado dos caprinos sem raça definida, mestiços e Anglo-nubiana pode ser explicado através das curvas ajustadas dos modelos gamma e polinomial quadrático. Os modelos gamma e polinomial quadrático apresentaram os melhores ajustes e os maiores poderes de explicação e menores valores de critério de informação de Akaike e soma dos quadrados dos resíduos independente da raça dos caprinos. Escolheu-se como modelo mais adequado para explicar o crescimento dos pesos dos caprinos, independente da raça, o gamma, pelo fato do mesmo apresentar um grau de explicação alto e menos parâmetros que o modelo polinomial quadrático.

\section{REFERÊNCIAS}

AKAIKE, $\mathrm{H}$. A new look at the statistical model identication. IEEE Transactions on automatic Control, v. 19, n. 6, p.716-723, 1974. Disponível em: $<$ http://ieeexplore.ieee.org/document/1100705> doi:10.1109/TAC.1974.1100705

CARNEIRO, P. L. S.; MALHADO, C. H. M.; AFFONSO, P. R. A. M.; PEREIRA, D. G.; SUZART, J. C. C.; JUNIOR, M. R.; ROCHA, J. L. Curva de crescimento em caprinos, da raça mambrina, criados na caatinga. Revista Brasileira de Saúde e Produção Animal, v. 10, p. 536-545, 2009.

AGRARIAN ACADEMY, Centro Científico Conhecer - Goiânia, v.4, n.7; p. 102017 
CAVALACANTE, D. H.; CAMPELO, J. E. G.; JÚNIOR, S. C. S.; SOUSA, G. G. T.; ARAUJO, J. I. M.; ARAUJO, A. C.; FONSECA, W. J. L.; JUNIOR, C. P. B.; ARAÚJO, A. M. Modelos não paramétricos para ajustes de curva de crescimento em caprinos sem raça definida (srd). Revista acadêmica: Ciências Agrárias e Ambiental, v. 11, p. 283-289, 2013. doi:10.7213/academica.011.003.AO08

CRUZ, G. R. B.; COSTA, R. G.; RIBEIRO, M. N. Curva de crescimento de caprinos mestiços no estado da Paraíba. Revista Brasileira de Ciências Agrárias, v. 4, n. 2, p. 204-210, 2009. Disponível em: <http://redalyc.uaemex/src/inicio/ArtPdfRed.jsp?iCve=119017351013>

DUTRA JR., W. M.; FERREIRA, A. S.; DONZELE, J. L.; EUCLYDES, R. F.; TAROUCO, J. U.; CARDOSO, L. L. Predição de curvas de crescimento de tecidos de fêmeas suínas por intermédio da função alométrica estendida. Revista Brasileira de Zootecnia, v. 30, n. 3, p. 007-1014, 2001. Disponível em: <http://dx.doi.org/10.1590/S1516-35982001000400014> doi:10.1590/S151635982001000400014

FERNANDES, T. L.; PEREIRA, A. A.; MUNIZ, J. A. Comparação de modelos nãolineares no estudo das curvas de crescimento do caranguejo de água doce. Revista da Estatística da Universidade Federal de Ouro Preto, v. 2, p. 375-2387, 2012.

FIGUEIREDO FILHO, L. A. S.; SARMENTO, J. L. R.; CAMPELO, J. E. G.; SANTOS, N. P. S.; SOUSA, J. E. R.; BIAGIOTTI, D. Fatores ambientais e genéticos sobre a curva de crescimento de caprinos mestiços. Comunicata Scientiae, v. 3, n. 3, p. 154-161, 2012.

FREITAS, A. R. Curvas de crescimento na produção animal. Revista Brasileira de Zootecnia, v. 34, n.3, p. 786-795, 2005. Disponível em: < http://dx.doi.org/10.1590/S1516-35982005000300010> doi:10.1590/S151635982005000300010

GADDOUR, A.; OUNI, M.; NAJARI, S. Growth curve estimation in pure goat breeds and crosses of first and second generation in Tunisian oases. Journal of Cell and Animal Biology, v. 6, n. 6, p. 99-103, 2012. Disponível em: < http://www.academicjournals.org/article/article1380281653_Gaddour\%20et\%20al.pdf > doi: $10.5897 / J C A B 12.018$

GBANGBOCHE, A. B.; GLELE-KAKAI, R.; SALIFOU, S.; ALBUQUERQUE, L. G.; LEROY, P. L. Comparison of non-linear growth models to describe the growth curve in west African dwarf sheep. Animal, v. 2, n. 7, p. 1003-1012, 2008. Disponível em: <https://www.ncbi.nlm.nih.gov/pubmed/22443700>doi:10.1017/S1751731108002206

MACEDO, L. R.; SILVA, F. F.; CIRILLO, M. A.; NASCIMENTO, M.; DÉBORA, M. P.; GUIMARÃES, S. E. F.; LOPES, P. S.; SANTOS, J. A.; AZEVEDO, C. F. Modelagem hierárquica Bayesiana na avaliação de curvas de crescimento de suínos AGRARIAN ACADEMY, Centro Científico Conhecer - Goiânia, v.4, n.7; p. 112017 
genotipados para o gene halotano. Ciência Rural, v. 44, n.10, p. 1853-1859, 2014. Disponível em: <http://dx.doi.org/10.1590/0103-8478cr20131278> doi:10.1590/01038478 cr20131278

MALHADO, C. H. M.; CARNEIRO, P. L. S.; CRUZ, J. F.; OLIVEIRA, D. F.; AZEVEDO, D. M. M. R.; SARMENTO, J. L. R. Curvas de crescimento para caprinos da raça anglo-nubiana criados na caatinga: rebanho de elite e comercial. Revista Brasileira de Saúde e Produção Animal, v. 9, n. 4, p. 662-671, 2008.

MORAIS, J.; FERREIRA, P. B.; JACONE, I. M. T. D.; MELLO, R.; BRENDA, F. C.; RORATO, P. R. N. Curva de crescimento de diferentes linhagens de frango de corte caipira. Ciência Rural, v. 45, n. 10, p. 1872-1878, 2015. Disponível em: < http://dx.doi.org/10.1590/0103-8478cr20130867> doi:10.1590/0103-8478cr20130867

Ó, A. L.; NETO, A. U. R.; SANTOS, G. V.; SARMENTO, J. L. R.; BIAGIOTTI, D.; SOUZA, J. E. R. Curva de crescimento de ovinos Santa Inês no vale do Gurgueial. Revista Brasileira de Saúde e Produção Animal, v. 13, n. 4, p. 912-922, 2012.

OLIVEIRA, L.; BRANDÃO, A. J. V.; BASSANEZI, R. C. Modelo de Von Bertalanffy generalizado aplicado ao crescimento de suínos de corte. Biomatemática, v. 17, p. 101-109, 2007.

OZDEMIR, H.; DELLAL, G. Determination of growth curves in young angora goats. Tarim Bilimleri Dergisi, v. 15, n. 4, p. 358-362, 2009.

SILVA, F. L.; ALENCAR, M. M.; FREITAS, A. R.; PACKER, I. U.; MOURÃO, G. B. Curvas de crescimento em vacas de corte de diferentes tipos biológicos. Pesquisa Agropecuária Brasileira, v. 46, n. 3, p. 262-271, 2011.

SOUZA, L. A.; CAIRES, D. N.; CARNEIRO, P. L. S.; MALHADO, C. H. M.; FILHO, R. $M$. Curvas de crescimento em bovinos da raça indubrasil criados no estado de Sergipe. Revista Ciência Agronômica, v. 41, n. 4, p. 671-676, 2010. Disponível em: <http://dx.doi.org/10.1590/S1806-66902010000400022> doi:10.1590/S180666902010000400022

SOUZA, L. A.; CARNEIRO, P. L. S.; MALHADO, C. H.; PAIVA, S. R.; CAIRES, D. N.; BARRETO, D. L. F. Curvas de crescimento em ovinos da raça morada nova criados no estado da Bahia. Revista Brasileira de Zootecnia, v. 40, n. 8, p. 1700-1705, 2011. Disponível em: <http://dx.doi.org/10.1590/S1516-35982011000800011> doi:10.1590/S1516-35982011000800011

TEXEIRA, M. C.; VILLARROEL, A. B.; PEREIRA, E. S.; OLIVEIRA, S. M. P.; ALBUQUERQUE, I. A.; MIZUBUTI, I. Y. Curva de crescimento de cordeiros de três sistemas de produção na região nordeste do Brasil. Semina: Ciências Agrárias, v. 33, n. 5, p. 2011-2018, 2012. Disponível em: < http://www.uel.br/revistas/uel/index.php/semagrarias/article/viewFile/9655/11580> doi:10.5433/1679-0359

VARGAS, G. D.; DIONELLO, N. L.; BRUM, P. A. R.; RUTZ, F.; FIALHO, F. B. 
Modelagem de crescimento e do desenvolvimento de frangos de corte: validação. Ciência Rural, v. 36, n. 5, p. 1664-1669, 2006. Disponível em: < http://dx.doi.org/10.1590/S0103-84782006000500053> doi:10.1590/S010384782006000500053

WAHEED, A.; KHAN, M. S.; ALI, A.; FE, M. S. Estimation of growth curve parameters in beetal goats. Archiv Tierzucht, v. 54, n. 3, p. 287-296, 2011. 